\title{
Stage IIIB Mycosis Fungoides and Sezary Syndrome AJCC v8
}

National Cancer Institute

\section{Source}

National Cancer Institute. Stage IIIB Mycosis Fungoides and Sezary Syndrome A/CC v8. NCI Thesaurus. Code C141355.

Stage IIIB includes: T4, N0-2, M0, B1. T4: Confluence of erythema covering $80 \%$ or more of body surface area. N0: No clinically abnormal peripheral lymph nodes; biopsy not required. N1: Clinically abnormal peripheral lymph nodes; histopathology Dutch grade 1 or National Cancer Institute (NCI) LNO-2. N2: Clinically abnormal peripheral lymph nodes; histopathology Dutch grade 2 or NCI LN3. M0: No visceral organ involvement. B1: Low blood tumor burden: more than $5 \%$ of peripheral blood lymphocytes are atypical (Sezary cells) but does not meet the criteria of B2. (AJCC 8th ed.) 